\title{
Atrophied brain lesion volume, a magnetic resonance imaging biomarker for monitoring neurodegenerative changes in multiple sclerosis
}

\author{
Robert Zivadinov $^{1,2}$, Niels Bergsland ${ }^{1}$, Michael G. Dwyer ${ }^{1}$ \\ ${ }^{1}$ Buffalo Neuroimaging Analysis Center, Department of Neurology, Jacobs School of Medicine and Biomedical Sciences, University at Buffalo, State \\ University of New York, Buffalo, NY, USA; ${ }^{2}$ Center for Biomedical Imaging, University at Buffalo, State University of New York, Buffalo, NY, USA \\ Correspondence to: Robert Zivadinov, MD, PhD. Center for Biomedical Imaging at Clinical Translational Science Institute, Jacobs School of Medicine \\ and Biomedical Sciences, University at Buffalo, State University of New York, 100 High Street, Buffalo, NY 14203, USA. Email: rzivadinov@bnac.net.
}

Submitted Oct 21, 2018. Accepted for publication Oct 31, 2018.

doi: 10.21037/qims.2018.11.01

View this article at: http://dx.doi.org/10.21037/qims.2018.11.01

Multiple sclerosis (MS) is a chronic, autoimmune disease of the central nervous system (CNS) that is characterized by inflammation, demyelination and axonal loss (1). Since its discovery, magnetic resonance imaging (MRI) has revolutionized the diagnosis and monitoring of patients with MS (2). Conventional MRI measures, like $\mathrm{T} 2$ hyperintense lesions on T2-weighted images (WI) and contrast enhancing (CE) lesions on post-contrast T1-WI are now routinely used to detect therapeutic effects and extend clinical observations (3). For example, accumulation of new or enlarging brain $\mathrm{T} 2$ hyperintense lesions or changes in hyperintense T2 lesion volume (LV) have been used as primary endpoints in phase II clinical trials, and as a secondary endpoint in phase III trials in MS (1-5). In addition, monitoring of the appearance of new lesions and the enlargement of existing ones is typically interpreted as an indicator of disease activity in the clinical routine (6). However, detection of $\mathrm{T} 2$ and $\mathrm{CE}$ lesion activity has insufficient specificity to reveal the true degree of pathological changes occurring in MS, as they cannot distinguish between inflammation, edema, demyelination, Wallerian degeneration, and axonal loss $(1,2)$. In addition to white matter (WM) lesions, MS pathology is characterized by focal demyelination in the gray matter (GM), cortical demyelination and diffuse axonal loss in normal appearing WM and GM (7). Because of these reasons, WM lesion accumulation correlates poorly with clinical evolution in the mid- to long-term (1,2,8-11), and more meaningful imaging biomarkers of neurodegeneration and clinical disease progression in MS patients are a topic of intense research (7,12-15).

Over the last two decades, enormous progress has been made in identifying new MRI biomarkers that are better reflective of neurodegenerative and destructive pathological processes related to disease activity and clinical progression in MS over time, compared to the conventional ones $(1,2,7)$. Among these, measurement of T1-weighted hypointense (16), cortical (17) and slowly expanding or smoldering (18) lesions, assessment of leptomeningeal enhancement by use of three-dimensional post-contrast fluid-attenuated inversion recovery (19), measurement of brain and spinal cord atrophy $(7,12,13)$, use of magnetization transfer imaging (20), myelin water fraction (21), diffusiontensor imaging (22), magnetic resonance spectroscopy (23), quantitative susceptibility mapping (24) and ultra-high field MRI (25), have been introduced as meaningful research tools to investigate severity of neurodegeneration and clinical disease progression in MS patients. However, the translation of these MRI biomarkers outside of research studies and clinical trials has been limited, as there are numerous technical and logistical hurdles to real-world implementation that must be overcome (7). Such challenges include the lack of non-conventional MRI protocols in many academic and private MS centers, frequent scanner, software, and protocol changes that limit standardization of the MRI measures longitudinally, and normal biological fluctuations that confound precise individual measurement $(26,27)$. 
In order for an MRI biomarker to be a useful tool to predict disability progression or determine conversion from relapsing to progressive disease stages, it must show an acceleration of the measured parameter several years before clinical disease progression or conversion occurs. However, most non-conventional MRI measures show steady linear changes over time and are not particularly dependent on changes in disease stage. For example, a recent study demonstrated that the rate of thalamic atrophy is consistent throughout the entire disease duration, regardless of MS clinical phenotype (28). While it is well known that patients who develop more severe GM atrophy have a higher chance to develop disability progression and conversion to progressive stages of the disease, it is currently impossible to reliably predict when this will occur early in the disease process (7). Moreover, with the advance of the disease and aging, the underlying pathology transitions towards a less inflammatory and more neurodegenerative course in which disease activity dramatically declines, especially in patients with longer disease duration and advanced disability. As a consequence, in this stage of the disease, MRI examination is not performed as regularly, because it is believed that disease may be "burned-out". This also leads frequently to discontinuation of treatment in older subjects without apparent clinical and radiological disease activity, which is a topic of intensive current debate in the scientific community (29).

Therefore, to develop MRI measures that can be obtained on clinical routine quality scans, that can consistently distinguish between multiple MS phenotypes, and that can determine early on which patients are at increased risk to develop disability progression, is highly warranted and currently lacking. The MS lesions located around central brain structures (e.g., ventricles) are particularly vulnerable to disease pathology, likely in part due cerebrospinal fluid (CSF)-mediated factors (15). Critically, though, instead of simply accumulating, lesions may also paradoxically shrink or disappear entirely due to atrophy. New and old lesions alike may be destroyed by atrophy and subsumed into the CSF. As a consequence, the full extent of lesion accumulation may be masked, and the $\mathrm{T} 2-\mathrm{LV}$ may even plateau or decline in the advanced stages of the disease, in the absence of any true resolution or repair (1). While generally neglected, this phenomenon may actually be an important stand-alone imaging biomarker. Recently introduced as "atrophied brain lesion volume" (Figure 1), this biomarker measures the precise volume of lesions disappearing into CSF spaces that are longitudinally replaced by the CSF or that are being displaced by substantial atrophy-related local movement at subsequent time points (15).

In a recent study, Dwyer et al. (15) investigated the rate of brain lesion loss due to atrophy (atrophied T2-LV) over 5 years, and evaluated its independent predictive value for the development of clinical disability in 174 MS and 18 patients with clinically isolated syndrome (CIS). Atrophied T2-LV was significantly different between disease stages, with progressive MS patients showing the highest rate of atrophied T2-LV, compared to those with relapsingremitting (RR) or CIS subtypes. Neither new nor enlarging T2-LVs were different between disease stages. When disability progression was related to MRI outcomes (new and enlarging T2-LV, brain atrophy and atrophied T2-LV), only atrophied T2-LV was associated with development of disability, and explained significant additional variance in predicting disability, even when controlling for both new/enlarging lesion activity and brain atrophy. Another recent study investigated temporal changes of cumulative atrophied brain T2-LV and 10-year confirmed disability progression in 176 investigated RRMS patients who obtained MRI examination at baseline, 6 months and then yearly over 10 years (30). Over 10 years, 76 patients remained stable and 100 developed confirmed disability progression. The cumulative atrophied T2-LV showed a robust association with development of 10-year confirmed disability progression, and this association was maintained across all time points with an effect size similar to that of whole brain atrophy. In addition, atrophied T2-LV was associated with the development of 10-year confirmed disability progression for almost all serial time points, which was not the case for whole brain atrophy, making atrophied T2-LV a potentially more attractive MRI outcome for clinical monitoring on a year-to-year clinical routine basis, in comparison to development of brain atrophy or lesion accrual. Atrophied T2-LV was also an earlier predictor of disability, as it took only 6 months to show an association with time to confirmed disability progression, whereas it took 2 years for whole brain atrophy to show a comparable effect. Furthermore, atrophied T2-LV accumulation in that study was accelerated from years 5 to 10 of the follow-up, whereas accumulation of total T2-LV de-accelerated in the same time period, which suggests that this MRI outcome could be of particular interest in monitoring the transition from relapsing to progressive stages of MS. In accordance with the previous study (15), atrophied T2-LV significantly added to the prediction of mid-term-disability, even when 

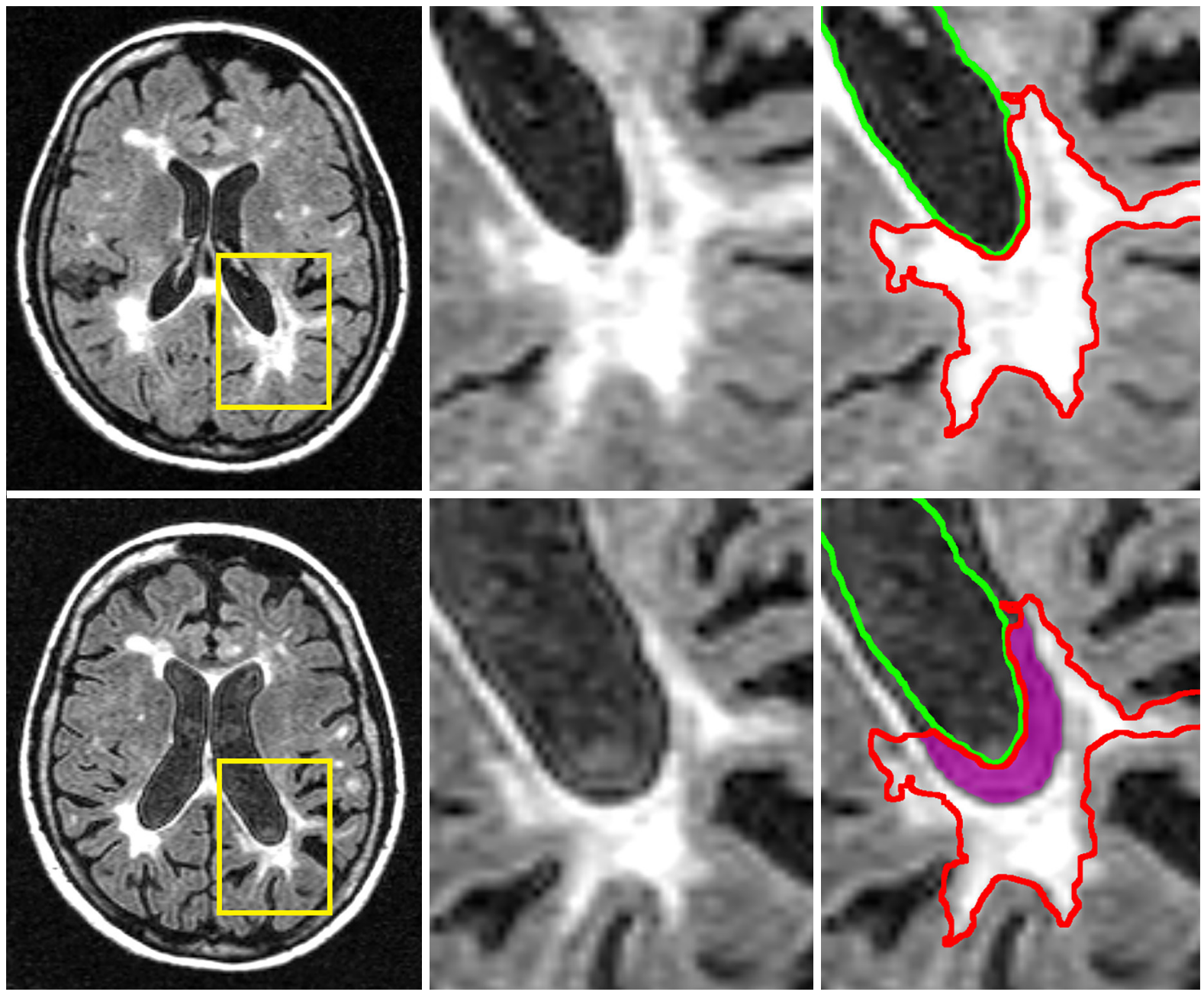

Figure 1 Representative example of periventricular (left) and non-periventricular (right) atrophied T2 lesion volume. Top row shows baseline T2-weighted fluid attenuated inversion recovery (T2-FLAIR), and bottom row shows follow-up T2-FLAIR. Left column shows original images, while center column shows an enlarged view of the inset areas indicated in yellow on the left. The right column replicates the center column, but with baseline lesion delineated in red and baseline ventricle border delineated in green. The delineations are identical at bottom right, to visualize the degree of change, with an additional magenta region showing the area that was lesion at baseline but has now been subsumed into cerebrospinal fluid (atrophied lesion).

accounting for the accumulation of new and enlarging $\mathrm{T} 2$ lesions and T2-LV, and development of brain atrophy. These data indicate that atrophying lesions are not a small secondary phenomenon in MS, and that they may play an increasingly important role as disease duration increases.

Given that atrophied T2-LV is a product of both inflammation (in the form of lesion accrual) and neurodegeneration (in the form of brain atrophy development), this MRI measure may add additional value in predicting disability progression or conversion to progressive disease stages than other MRI measures reflecting only a single aspect of pathophysiological progression. Although atrophied T2-LV represents only a small fraction of whole brain tissue loss $(15,30)$, its spatial and pathological specificity for tissue destruction may contribute to a wide range of neurological functions that may clinically translate into significant cognitive and physical disability. The clinical relevance of atrophied T2-LV could be related to the fact that the majority of the axons responsible for development of functional disability in MS patients are located around ventricles. However, because atrophied T2-LV also occurs at the gyri borders which atrophied to displace sulci CSF 
and replace parts of the lesions (15), it may also be related to cognitive impairment and other higher cortical functions. As the detection of cortical lesions remains challenging $(17,19,25)$, the true prevalence of atrophied T2-LV in cortical regions may be largely underestimated.

Atrophied T2-LV is a robust and early marker of disability progression in relapsing and progressive disease stages. Its clinical value has to be further explored in longitudinal, multi-center, double-blind, controlled and clinical routine studies using various disease-modifying treatments.

\section{Acknowledgements}

None.

\section{Footnote}

Conflicts of Interest: R Zivadinov received personal compensation from EMD Serono, Genzyme-Sanofi, Claret Medical, Celgene and Novartis for speaking and consultant fees. He received financial support for research activities from Genzyme-Sanofi, Novartis, Claret Medical, IntekrinCoherus, Protembis and Qunitiles/IMS. The other authors have no conflicts of interest to declare.

\section{References}

1. Barkhof $\mathrm{F}$. The clinico-radiological paradox in multiple sclerosis revisited. Curr Opin Neurol 2002;15:239-45.

2. Poloni G, Minagar A, Haacke EM, Zivadinov R. Recent developments in imaging of multiple sclerosis. Neurologist 2011;17:185-204.

3. Wattjes MP, Rovira A, Miller D, Yousry TA, Sormani MP, de Stefano MP, Tintore M, Auger C, Tur C, Filippi M, Rocca MA, Fazekas F, Kappos L, Polman C, Frederik B, Xavier M, group Ms. Evidence-based guidelines: MAGNIMS consensus guidelines on the use of MRI in multiple sclerosis--establishing disease prognosis and monitoring patients. Nat Rev Neurol 2015;11:597-606.

4. Bar-Zohar D, Agosta F, Goldstaub D, Filippi M. Magnetic resonance imaging metrics and their correlation with clinical outcomes in multiple sclerosis: a review of the literature and future perspectives. Mult Scler 2008;14:719-27.

5. Sormani MP, Bruzzi P, Comi G, Filippi M. MRI metrics as surrogate markers for clinical relapse rate in relapsingremitting MS patients. Neurology 2002;58:417-21.
6. Zivadinov R, Khan N, Medin J, Christoffersen P, Price J, Korn JR, Bonzani I, Dwyer MG, Bergsland N, Carl E, Silva D, Weinstock-Guttman B. An Observational Study to Assess Brain MRI Change and Disease Progression in Multiple Sclerosis Clinical Practice-The MS-MRIUS Study. J Neuroimaging 2017;27:339-47.

7. Zivadinov R, Jakimovski D, Gandhi S, Ahmed R, Dwyer MG, Horakova D, Weinstock-Guttman B, Benedict RR, Vaneckova M, Barnett M, Bergsland N. Clinical relevance of brain atrophy assessment in multiple sclerosis. Implications for its use in a clinical routine. Expert Rev Neurother 2016;16:777-93.

8. O'Riordan JI, Thompson AJ, Kingsley DP, MacManus DG, Kendall BE, Rudge P, McDonald WI, Miller DH. The prognostic value of brain MRI in clinically isolated syndromes of the CNS. A 10-year follow-up. Brain 1998; 121:495-503.

9. Rudick RA, Lee JC, Simon J, Fisher E. Significance of T2 lesions in multiple sclerosis: A 13-year longitudinal study. Ann Neurol 2006;60:236-42.

10. Sailer M, Losseff NA, Wang L, Gawne-Cain ML, Thompson AJ, Miller DH. T1 lesion load and cerebral atrophy as a marker for clinical progression in patients with multiple sclerosis. A prospective 18 months follow-up study. Eur J Neurol 2001;8:37-42.

11. Zivadinov R, Uher T, Hagemeier J, Vaneckova M, Ramasamy DP, Tyblova M, Bergsland N, Seidl Z, Dwyer MG, Krasensky J, Havrdova E, Horakova D. A serial 10-year follow-up study of brain atrophy and disability progression in RRMS patients. Mult Scler 2016;22:1709-18.

12. Bermel RA, Bakshi R. The measurement and clinical relevance of brain atrophy in multiple sclerosis. Lancet Neurol 2006;5:158-70.

13. De Stefano N, Airas L, Grigoriadis N, Mattle HP, O'Riordan J, Oreja-Guevara C, Sellebjerg F, Stankoff B, Walczak A, Wiendl H, Kieseier BC. Clinical relevance of brain volume measures in multiple sclerosis. CNS Drugs 2014;28:147-56.

14. Miller DH, Barkhof F, Frank JA, Parker GJ, Thompson AJ. Measurement of atrophy in multiple sclerosis: pathological basis, methodological aspects and clinical relevance. Brain 2002;125:1676-95.

15. Dwyer MG, Bergsland N, Ramasamy DP, Jakimovski D, Weinstock-Guttman B, Zivadinov R. Atrophied Brain Lesion Volume: A New Imaging Biomarker in Multiple Sclerosis. J Neuroimaging 2018;28:490-5.

16. van Walderveen MA, Barkhof F, Pouwels PJ, van Schijndel 
RA, Polman CH, Castelijns JA. Neuronal damage in T1hypointense multiple sclerosis lesions demonstrated in vivo using proton magnetic resonance spectroscopy. Ann Neurol 1999;46:79-87.

17. Geurts JJ, Pouwels PJ, Uitdehaag BM, Polman CH, Barkhof F, Castelijns JA. Intracortical lesions in multiple sclerosis: improved detection with 3D double inversionrecovery MR imaging. Radiology 2005;236:254-60.

18. Frischer JM, Weigand SD, Guo Y, Kale N, Parisi JE, Pirko I, Mandrekar J, Bramow S, Metz I, Bruck W, Lassmann $\mathrm{H}$, Lucchinetti CF. Clinical and pathological insights into the dynamic nature of the white matter multiple sclerosis plaque. Ann Neurol 2015;78:710-21.

19. Zurawski J, Lassmann H, Bakshi R. Use of Magnetic Resonance Imaging to Visualize Leptomeningeal Inflammation in Patients With Multiple Sclerosis: A Review. JAMA Neurol 2017;74:100-9.

20. Ropele S, Fazekas F. Magnetization Transfer MR Imaging in Multiple Sclerosis. Neuroimaging Clin N Am 2009;19:27-36.

21. Laule C, Yung A, Pavolva V, Bohnet B, Kozlowski P, Hashimoto SA, Yip S, Li DK, Moore GW. Highresolution myelin water imaging in post-mortem multiple sclerosis spinal cord: A case report. Mult Scler 2016;22:1485-9.

22. Velly L, Perlbarg V, Boulier T, Adam N, Delphine S, Luyt CE, Battisti V, Torkomian G, Arbelot C, Chabanne R, Jean B, Di Perri C, Laureys S, Citerio G, Vargiolu A, Rohaut B, Bruder N, Girard N, Silva S, Cottenceau V, Tourdias T, Coulon O, Riou B, Naccache L, Gupta R, Benali H, Galanaud D, Puybasset L, Investigators MC. Use of brain diffusion tensor imaging for the prediction of long-term neurological outcomes in patients after cardiac arrest: a multicentre, international, prospective, observational, cohort study. Lancet Neurol 2018;17:317-26.

23. Kirov, II, Liu S, Tal A, Wu WE, Davitz MS, Babb JS, Rusinek H, Herbert J, Gonen O. Proton MR spectroscopy of lesion evolution in multiple sclerosis: Steady-state metabolism and its relationship to conventional imaging. Hum Brain Mapp 2017;38:4047-63.

24. Zivadinov R, Tavazzi E, Bergsland N, Hagemeier J, Lin F,

Cite this article as: Zivadinov R, Bergsland N, Dwyer MG. Atrophied brain lesion volume, a magnetic resonance imaging biomarker for monitoring neurodegenerative changes in multiple sclerosis. Quant Imaging Med Surg 2018;8(10):979983. doi: 10.21037/qims.2018.11.01
Dwyer MG, Carl E, Kolb C, Hojnacki D, Ramasamy D, Durfee J, Weinstock-Guttman B, Schweser F. Brain Iron at Quantitative MRI Is Associated with Disability in Multiple Sclerosis. Radiology 2018;289:487-96.

25. Mainero C, Louapre C, Govindarajan ST, Gianni C, Nielsen AS, Cohen-Adad J, Sloane J, Kinkel RP. A gradient in cortical pathology in multiple sclerosis by in vivo quantitative 7 T imaging. Brain 2015;138:932-45.

26. Zivadinov R, Bergsland N, Korn JR, Dwyer MG, Khan N, Medin J, Price JC, Weinstock-Guttman B, Silva D, Group M-MS. Feasibility of Brain Atrophy Measurement in Clinical Routine without Prior Standardization of the MRI Protocol: Results from MS-MRIUS, a Longitudinal Observational, Multicenter Real-World Outcome Study in Patients with Relapsing-Remitting MS. AJNR Am J Neuroradiol 2018;39:289-95.

27. Zivadinov R, Khan N, Korn JR, Lathi E, Silversteen J, Calkwood J, Kolodny S, Silva D, Medin J, WeinstockGuttman B, group M-Ms. No evidence of disease activity in patients receiving fingolimod at private or academic centers in clinical practice: a retrospective analysis of the multiple sclerosis, clinical, and magnetic resonance imaging outcomes in the USA (MS-MRIUS) study. Curr Med Res Opin 2018;34:1431-40.

28. Azevedo CJ, Cen SY, Khadka S, Liu S, Kornak J, Shi Y, Zheng L, Hauser SL, Pelletier D. Thalamic atrophy in multiple sclerosis: A magnetic resonance imaging marker of neurodegeneration throughout disease. Ann Neurol 2018;83:223-34.

29. Kister I, Spelman T, Alroughani R, Lechner-Scott J, Duquette P, Grand'Maison F, Slee M, Lugaresi A, Barnett M, Grammond P, Iuliano G, Hupperts R, Pucci E, Trojano M, Butzkueven H, Group MSS. Discontinuing diseasemodifying therapy in MS after a prolonged relapsefree period: a propensity score-matched study. J Neurol Neurosurg Psychiatry 2016;87:1133-7.

30. Zivadinov R, Bergsland N, Hagemeier J, Ramasamy D, Uher T, Vaneckova M, Havrdova E, Horakova D, Dwyer M. Atrophied lesion volume: A novel and robust imaging biomarker in multiple sclerosis for predicting disability progression (P3.346). Neurology 2018;90. 